\title{
Estado y problemas de digitalización de la gestión de universidades en Rusia y en tres países latinoamericanos (Argentina, Chile y Brasil)
}

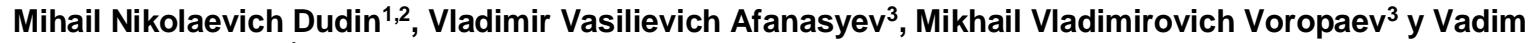 \\ Nikolaevich Zasko ${ }^{4}$ \\ (1) Market Economy Institute of RAS (MEI RAS), Nakhimovsky Prospect, 47, Moscow, 117418, Russian Federation \\ (2) Russian Presidential Academy of National Economy and Public Administration (RANEPA), Vernadsky Prospect, 82 , \\ Moscow, 119571, Russian Federation (correo-e: dudinmn@mail.ru) \\ (3) Moscow City University, Vtoroy Selskohoziajstvenny Proezd, 4, Moscow, 129226, Russian Federation \\ (4) Financial University under the Government of the Russian Federation, Leningradsky Prospect, 49, Moscow, 125993, \\ Russian Federation
}

Recibido Feb. 17, 2020; Aceptado Abr. 17, 2020; Versión final Jun. 24, 2020, Publicado Dic. 2020

\begin{abstract}
Resumen
El propósito de esta investigación es estudiar la calidad de la digitalización gerencial y de las funciones vinculadas a ésta en universidades de Rusia, Brasil, Chile y Argentina. En el trabajo participaron 65 expertos, cuyo principal lugar de trabajo son universidades de estos países. Los datos obtenidos permiten afirmar que, en primer lugar, la eficacia de los sistemas nacionales de innovación en Rusia, Brasil, Chile y Argentina no es alta. En segundo lugar, la calidad de la digitalización de la gestión universitaria en estos países se encuentra en un nivel medio; esto se debe al hecho de que el aparato directivo y el personal docente de las universidades no son lo suficientemente competentes y existe un claro problema de financiación de la digitalización. Se concluye que existe una relación objetiva entre el nivel de sofisticación de los sistemas nacionales de innovación y la calidad de la digitalización de las funciones de gestión universitaria.
\end{abstract}

\section{Situation and problems of digitation of university management in Russia and in three Latin-American countries (Argentina, Chile, and Brazil)}

\begin{abstract}
The purpose of this research study is to assess the digitization management quality and related functions in universities from Russia, Brazil, Chile, and Argentina. The study participants were 65 experts working at the selected universities. The results show that there is low efficiency in the national innovation systems from Russia, Brazil, Chile and Argentina. In addition, digitization quality for university management is at an average level in all of these countries. This may be due to the fact that management and teaching staff from the universities examined are not sufficiently competent. In addition, there is an evident problem with digitization funding. It is concluded that there is a correlation between the sophistication of national innovation systems and the digitization quality of university management functions.
\end{abstract}

Keywords: digital technologies; university management digitization; triple helix model 


\section{INTRODUCCIÓN}

Las palabras atribuidas al gran pensador chino Confucio hablan de la complejidad de la vida en una era de cambio, sin embargo, el poeta ruso $\mathrm{F}$. Tyutchev creía que el momento del cambio, es quizás el mejor momento para articular nuevos planes e implementar nuevas ideas. Por lo tanto, creemos que el presente, probablemente sea el momento donde ocurran los mayores cambios tecnológicos en la historia moderna de la humanidad, y se abran nuevas oportunidades para mejorar el funcionamiento de muchas áreas, tanto tecnológicas como sociales, para proporcionar al futuro de todos, nuevos conocimientos, en particular a través de la renovación del sector científico y educativo. En este contexto son vitales las ideas, que permiten en primer lugar, actualizar cualitativamente la función de gestión de las universidades, que son productoras de nuevos conocimientos, que estimulan la velocidad del progreso científico y tecnológico en el mundo.

Las tecnologías digitales que se pueden utilizar para mejorar las funciones de gestión de universidades, abren nuevas posibilidades, pero al mismo tiempo, el uso de estas tecnologías en este campo tiene ciertas limitaciones. Por lo tanto, por un lado, observamos objetivamente la necesidad de digitalizar las funciones de gestión de las universidades, pero, por otro lado, nos enfrentamos al hecho de que, en algunas organizaciones científicas y educativas, e incluso en algunos sistemas científicos y educativos nacionales, existen ciertas reticencias por parte del profesorado, de los funcionarios responsables de la administración de los sistemas educativos (Bogoviz et al., 2018; Maymina et al., 2018; Ordorika y Rodríguez-Gómez, 2018). Es obvio que la digitalización de las funciones de gestión universitaria (por supuesto, en conjunto con otras funciones) y el problema de digitalización de las instituciones de educación superior, son un problema actual que requiere la realización de investigaciones en esta dirección. En general, se acepta que los líderes en la digitalización de la gestión, la investigación, la enseñanza (educación) y otras funciones son universidades en los Estados Unidos y algunos países de la Unión Europea.

La razón principal es el alto nivel de desarrollo socioeconómico, que permite utilizar los efectos positivos de la fusión del capital privado, el conocimiento y el apoyo estatal para superar el progreso científico, técnico y tecnológico de estos países, lo que, en particular, permitió a H. Etzkowitz en colaboración con L. Leydesdorff (1996) y C. Zhou (2017) presentar la hipótesis de la triple hélice, que sirve como medio para la formación de sistemas nacionales de innovación altamente productivos.Podemos decir que el modelo de la triple hélice está completamente implementado en los Estados Unidos (Silicon Valley); con acentuadas características nacionales, pusieron en práctica este modelo también Japón, Canadá, algunos países de Europa Occidental e incluso Bielorrusia (Slobodskaya, 2018). En Rusia, Argentina, Brasil y Chile también se han implementado proyectos que utilizan el concepto de la triple hélice para crear sistemas nacionales de innovación. Pero, como demuestra la práctica, las economías de estos tres países aún se encuentran en desarrollo, y los sectores universitarios y, en general, científicos-educativos y de investigación se caracterizan por un menor nivel de digitalización, que puede ser el factor que obstaculice el progreso técnico y tecnológico en Rusia. Argentina, Chile y Brasil.

Por lo tanto, la hipótesis de nuestra investigación es la afirmación de que la digitalización gerencial y por ende, otras funciones de las actividades universitarias pueden contribuir a la formación de un sistema nacional de innovación efectivo, basado en la unificación del estado, el sector empresarial y la ciencia, para lograr un desarrollo socioeconómico dinámicamente sostenible de acuerdo con una comprensión avanzada de tal desarrollo, establecida en documentos de la ONU (United Nations, 2020). En consecuencia, como objetivos de nuestro estudio determinamos: en primer lugar, el análisis de contenido de fuentes científicas con respecto a las funciones de las actividades universitarias y a la posibilidad, la necesidad y la viabilidad de digitalizar estas funciones; en segundo lugar, el análisis de datos estáticos sobre la dinámica de digitalización de diversas funciones en universidades de Rusia, Brasil, Chile, Argentina, así como del estado y nivel de sofisticación de los sistemas nacionales de innovación de estos países; en tercer lugar, la evaluación de la calidad de la digitalización de las funciones de gestión en universidades de Rusia, Brasil, Chile, Argentina, utilizando métodos especializados y algoritmos difusos múltiples; en cuarto lugar, el estudio comparativo de las limitaciones que no permiten mejorar las funciones de gestión de las universidades en Rusia, Brasil, Chile y Argentina mediante el uso de tecnologías digitales. La novedad científica del artículo radica en el estudio de la influencia mutua de dos factores (la digitalización de las universidades y el desarrollo del sistema nacional de innovación) en el contexto de la calidad y la dinámica del desarrollo socioeconómico de Rusia, Brasil, Chile y Argentina.

\section{OTROS ANTECEDENTES}

Numerosos estudios realizados, incluso sobre materiales de universidades (Legner et al., 2017; Strack et al., 2017; Dudin et al., 2018; Vuori et al., 2019; United Nations, 2020) indican que la automatización y la digitalización actúan como herramientas para garantizar los intereses de estabilidad y desarrollo de las instituciones educativas, así como para disminuir los riesgos inherentes a ellas. En este sentido, y teniendo 
en cuenta la transformación digital de los procesos socioeconómicos observada en la última década, en el contexto de garantizar el funcionamiento estable y el desarrollo dinámico de las universidades, va adquiriendo cada vez mayor relevancia el tema del uso de las tecnologías digitales modernas en la gestión.

En primer lugar, es necesario determinar el aparato terminológico para que la presentación posterior de los materiales de investigación sea lógicamente consistente. Los términos más importantes que se deben especificar son: tecnologías digitales; funciones de gestión universitaria; otras funciones de actividades universitarias; sistema nacional de innovación. Por lo tanto, las tecnologías digitales son una clase de tecnologías que permite transmitir información y señales de forma discreta, lo que ayuda a eliminar las distorsiones al transmitir un flujo de información o un flujo de señal; para dicha transmisión, por regla general, se utilizan dispositivos y equipos digitales especiales (desde computación electrónica digital hasta sistemas de ciberfísica). La literatura científica moderna nos permite distinguir dos grupos de tecnologías digitales (Tabla 1), que, entre otros, se pueden utilizar para mejorar las funciones de gestión universitaria (u otras funciones de las actividades universitarias).

Tabla 1: Clasificación de las tecnologías digitales (Brennen y Kreiss, 2016; Ng, 2016)

\begin{tabular}{|l|l|}
\hline Clases de tecnologías digitales del presente & Clases de tecnologías digitales del futuro \\
\hline $\begin{array}{l}\text { Tecnologías de la información, la comunicación y la } \\
\text { informática. }\end{array}$ & $\begin{array}{l}\text { Internet de las Cosas (así como el Internet Industrial de } \\
\text { las cosas) }\end{array}$ \\
\hline $\begin{array}{l}\text { Tecnologías de apoyo en la toma de decisiones, } \\
\text { incluidas las de gestión, finanzas, logística y otras } \\
\text { tecnologías de servicios }\end{array}$ & Realidad virtual y realidad aumentada \\
\hline $\begin{array}{l}\text { Tecnologías digitales de precisión para diversas } \\
\text { actividades económicas }\end{array}$ & $\begin{array}{l}\text { Aprendizaje automático e inteligencia artificial (agentes } \\
\text { intelectuales) }\end{array}$ \\
\hline Tecnologías digitales, orientadas al sector social & $\begin{array}{l}\text { Robótica (sistemas ciberfísicos) y tecnologías aditivas } \\
\text { (impresión 3D) }\end{array}$ \\
\hline
\end{tabular}

Consideramos que la digitalización de la gestión universitaria, así como la digitalización de otras funciones de las actividades universitarias en las condiciones modernas, deberían incorporar plenamente la clase de tecnologías del presente y, hacer todo lo posible por lograr el paso al uso de tecnologías del futuro. A su vez, consideramos correcto combinar las funciones que posibilitan las actividades universitarias en tres grupos principales, Tabla 2.

Tabla 2: Agrupación de funciones que posibilitan las actividades (funcionamiento y desarrollo) de las universidades [compiladas por los autores]

\begin{tabular}{|l|l|l|}
\hline $\begin{array}{l}\text { Funciones administrativas y } \\
\text { empresariales de gestión }\end{array}$ & $\begin{array}{l}\text { Funciones educativas y de } \\
\text { investigación. }\end{array}$ & Funciones sociales \\
\hline $\begin{array}{l}\text { Organización, planificación y gestión } \\
\text { de procesos dirigidos (formación de } \\
\text { estudiantes, investigaciones } \\
\text { científicas) }\end{array}$ & $\begin{array}{l}\text { Creación y realización de } \\
\text { programas de formación científica } \\
\text { para los estudiantes en } \\
\text { correspondencia con la } \\
\text { especialización de la universidad }\end{array}$ & $\begin{array}{l}\text { Formación y desarrollo de recursos } \\
\text { laborales altamente profesionales, de } \\
\text { gran demanda en la economía } \\
\text { nacional y el estado }\end{array}$ \\
\hline $\begin{array}{l}\text { Interacción con partes interesadas } \\
\text { gobierno, empresarios, otros grupos } \\
\text { de referencia) e inversores }\end{array}$ & $\begin{array}{l}\text { Realización de investigaciones y } \\
\text { estudios sobre la especialización de } \\
\text { la universidad, de acuerdo con las } \\
\text { tareas estatales, etc. }\end{array}$ & $\begin{array}{l}\text { Garantía de la transferencia y difusión } \\
\text { de ideas científicas, tecnologías, etc. } \\
\text { En la sociedad, incluidas la actividad } \\
\text { científica y educativa }\end{array}$ \\
\hline $\begin{array}{l}\text { Garantizar la transparencia de la } \\
\text { universidad y la creación de las } \\
\text { condiciones para la conversión de los } \\
\text { resultados de la actividad intelectual } \\
\text { al sector real, financiero y social. }\end{array}$ & $\begin{array}{l}\text { Cooperación científica, técnica y } \\
\text { tecnológica con el sector científico y y } \\
\text { no científico. }\end{array}$ & $\begin{array}{l}\text { Desarrollo de tecnologías e ideas } \\
\text { para garantizar las responsabilidades } \\
\text { ambientales y de otro tipo de la } \\
\text { generación actual ante las } \\
\text { generaciones futuras. }\end{array}$ \\
\hline
\end{tabular}

El sistema nacional de innovación es, en esencia, la triple espiral que une y fusiona los intereses del estado, el emprendimiento (negocios) y las universidades (en sentido amplio, o sea, como los sectores de investigación y educación) en el campo de la sociedad dinámicamente sostenible, con un desarrollo económico basado en el uso efectivo e intensivo de los conocimientos fundamentales y aplicados en la práctica (Godin, 2009; Etzkowitz y Zhou, 2017). Para pasar a una forma digital de gestión, es necesario realizar una transformación digital de la universidad, que implica el uso de todo el arsenal de tecnologías digitales modernas de información y comunicación para aumentar drásticamente la productividad y el valor de los servicios educativos, estimular el proceso científico-técnico y tecnológico en la economía, el sector social y cumplir otras funciones sociales y comercialmente significativas. 
Si anteriormente, en términos de automatización de la gestión de universidades, solo se consideraba la optimización de fases individuales o etapas de gestión (Hattinger et al., 2018), hoy, en el marco de la transformación digital y la creación de una universidad digital, se analiza un proceso continuo que incluye no solo las etapas de producción, desde la idea, el desarrollo, el diseño, la provisión y hasta la prestación de servicios educativos, sino también actividades empresariales y financieras relacionadas, trabajo del personal, logística, gestión, soporte, Y red asociada,

Debe ser tenido en cuenta que: en primer lugar, cualquier decisión de digitalización debe tener argumentación económica, en algunos casos puede ser aconsejable seguir la instrumentación de gestión tradicional, especialmente porque las finanzas son la limitación más clásica en el desarrollo de la universidad; en segundo lugar, las decisiones deben tener en cuenta el aspecto social. Hasta ahora, los teóricos y prácticos de la digitalización han estado discutiendo sobre la mejor manera de resolver el problema de liberar a los empleados cuyos lugares han sido digitalizados. El argumento común de que estas personas se convertirán en operadores condicionales de computadores digitales no tiene una relación real con la solución del problema: no hay posibilidad de emplear ni siquiera una cuarta parte de los trabajadores liberados, además de que no todos están listos para convertirse en operadores de computadoras, teniendo en cuenta el nivel de formación profesional y requisitos salariales. En pocas palabras, para controlar de forma calificada el computador, prácticamente no tiene importancia quién estará al panel del operador: el académico o el asistente de laboratorio. Y las necesidades financieras de estos trabajadores serán diferentes; en tercer lugar, Es importante tener en cuenta los riesgos tecnológicos y de otro tipo: los sistemas de información se caracterizan por numerosas vulnerabilidades y, a menudo, las soluciones requieren duplicación y otras medidas de seguridad; en cuarto lugar, No deben tratar de ser pioneros de la digitalización, al menos las universidades que no se especializan en tecnologías de la información. Ni en términos de conocimiento, ni en la suficiencia de los recursos financieros, son adecuadas para este papel. En nuestra opinión, la implementación de proyectos de digitalización para la gestión de universidades debe ser gradual y planificarse racionalmente.

\section{MATERIALES Y MÉTODOS}

Para analizar las prácticas y problemas de la digitalización de la gestión universitaria usamos los siguientes métodos de investigación: análisis estadístico, que nos permite rastrear la dinámica del desarrollo socioeconómico e innovador de los países estudiados (Rusia, Brasil, Argentina, Chile); análisis de la digitalización de las funciones de gestión universitaria (así como la digitalización de otras áreas funcionales de la actividad universitaria) mediante la recopilación de opiniones de expertos y la evaluación de su coherencia utilizando el coeficiente de concordancia; evaluación de la calidad de la digitalización de las funciones de gestión en las actividades de las universidades de Rusia, Brasil, Chile, Argentina utilizando un modelo especialmente desarrollado, que se basa en funciones matemáticas múltiples difusas.

La recopilación de opiniones de expertos se llevó a cabo de la siguiente forma: en primer lugar, se desarrolló un modelo que incluía juicios de valor de expertos sobre cinco parámetros principales (Tabla 3 ) de la digitalización de funciones de gestión universitaria; en segundo lugar, los juicios de expertos recopilados se analizaron para determinar su consistencia utilizando herramientas especiales (Fórmula 1); en tercer lugar, las opiniones de expertos obtenidas se transformaron en una evaluación de la digitalización de las funciones de gestión, utilizando un algoritmo difuso múltiple en el entorno MathCad (Tablas 4 y 5); en cuarto lugar, el valor final del nivel de digitalización de las funciones gerenciales se obtuvo usando una función matemática ponderada (Fórmulas 2 y 3), los criterios ponderados de la función se calcularon usando el método Fishburn (Fórmula 4).

Tabla 3: Parámetros para la investigación de la digitalización de funciones de gestión universitaria [elaborado por los autores]

\begin{tabular}{|c|c|c|c|c|c|}
\hline \multirow[b]{2}{*}{ Parámetros } & \multicolumn{5}{|c|}{ Valor de parámetro estimado por expertos } \\
\hline & mínimo & $\begin{array}{l}\text { inferior a la } \\
\text { media }\end{array}$ & medio & superior a la media & máximo \\
\hline $\begin{array}{l}\text { Competencias necesarias para la digitalización } \\
\text { de la gestión universitaria, } x_{1}\end{array}$ & \multicolumn{5}{|c|}{$\begin{array}{l}\text { Suficiencia de las competencias generales y especiales de la administración universitaria y } \\
\text { del profesorado superior para el desempeño de sus funciones utilizando tecnologías } \\
\text { digitales. }\end{array}$} \\
\hline tión u & \multicolumn{5}{|c|}{$\begin{array}{l}\text { Provisión de funciones gerenciales y otras relacionadas y requeridas por herramientas } \\
\text { técnicas y tecnológicas, equipos para el desempeño de estas funciones en modo digital }\end{array}$} \\
\hline $\begin{array}{l}\text { Voluntad de realizar cambios: tra } \\
\text { digitalización de la gestión univer }\end{array}$ & \multicolumn{5}{|c|}{$\begin{array}{l}\text { Buena disposición del personal directivo de las universidades y del profesorado superior, es } \\
\text { decir, la voluntad de usar tecnología digital para las obligaciones propias del cargo }\end{array}$} \\
\hline $\begin{array}{l}\text { Financiamiento de la digitalización de la gestión } \\
\text { universitaria, } x_{4}\end{array}$ & \multicolumn{5}{|c|}{$\begin{array}{l}\text { Suficiencia de recursos financieros (en los fondos universitarios o en el presupuesto } \\
\text { departamental) para la digitalización material y tecnológica de las funciones de gestión (y } \\
\text { funciones interrelacionadas) }\end{array}$} \\
\hline $\begin{array}{l}\text { Beneficios y perspectivas de la digitalización de } \\
\text { la gestión universitaria, } x_{5}\end{array}$ & \multicolumn{5}{|c|}{$\begin{array}{l}\text { Opinión del personal de gestión de las universidades y el profesorado superior sobre las } \\
\text { oportunidades, ventajas y perspectivas que su universidad puede obtener al hacer la } \\
\text { transición a las tecnologías digitales en la gestión y otras áreas funcionales }\end{array}$} \\
\hline
\end{tabular}


El coeficiente de concordancia, es decir, la consistencia de las opiniones de expertos se calculó utilizando la siguiente fórmula:

$$
\begin{aligned}
W & =\frac{S}{0.083 * m^{3} *\left(n^{3}-n\right)-m * \sum T_{i}} \\
\chi^{2} & =\frac{S}{0.083 * m * n *(n+1)+1 / n-1} m * \sum T_{i}
\end{aligned}
$$

En estas ecuaciones $W$ y $\chi$ es en consecuencia, el coeficiente de concordancia y el criterio de correspondencia de Pearson; $S$ es el cuadrado del número de control en la matriz de filas de opiniones de expertos; $m$ es la cantidad de integrantes del grupo de expertos; $n$ es la cantidad de parámetros de la evaluación hecha por los expertos; $T_{i}$ es la coherencia de opinión del experto número-i

Tabla 4: Análisis de la pertenencia de los parámetros de digitalización individuales de las funciones de gestión universitaria a

\begin{tabular}{|c|c|c|}
\hline $\begin{array}{l}\text { Serie de valores de } \\
\text { variables (parámetros) } \\
\text { incluidos en el modelo }\end{array}$ & $\begin{array}{l}\text { Descripción de los subconjuntos de variables } \\
\text { (parámetros) incluidos en el modelo, en términos } \\
\text { de calidad de digitalización. }\end{array}$ & $\begin{array}{l}\text { Función que describe la } \\
\text { pertenencia de un valor variable a } \\
\text { un subconjunto }\end{array}$ \\
\hline $0<x_{i}<0,1$ & \multirow{2}{*}{$\begin{array}{l}\text { Valor mínimo de la variable, muy baja calidad de } \\
\text { digitalización }\end{array}$} & $f=1$ \\
\hline $0,1<x_{i}<0,15$ & & $f_{1}=10 *\left(0,15-x_{i}\right)$ \\
\hline $0,1<x_{i}<0,15$ & \multirow{3}{*}{$\begin{array}{l}\text { Valor bajo de la variable, mala calidad de } \\
\text { digitalización }\end{array}$} & $f_{2}=1-f_{1}$ \\
\hline $0,15<x_{i}<0,25$ & & $f=1$ \\
\hline $0,25<x_{i}<0,35$ & & $f_{2}=10 *\left(0,35-x_{i}\right)$ \\
\hline $0,25<x_{i}<0,35$ & \multirow{3}{*}{$\begin{array}{l}\text { Valor de la variable no superior a la media, calidad } \\
\text { de digitalización promedio }\end{array}$} & $f_{3}=1-f_{2}$ \\
\hline $0,35<x_{i}<0,45$ & & $f=1$ \\
\hline $0,45<x_{i}<0,55$ & & $f_{3}=10 *\left(0,55-x_{i}\right)$ \\
\hline $0,45<x_{i}<0,55$ & $\begin{array}{l}\text { Valor de la variable promedio y superior al } \\
\text { promedio; calidad de digitalización superior al } \\
\text { promedio }\end{array}$ & $f_{4}=1-f_{3}$ \\
\hline $0,5<x_{i}<0,6$ & \multirow{2}{*}{$\begin{array}{l}\text { Valor de la variable superior al promedio y de un } \\
\text { valor alto, alta calidad de la digitalización }\end{array}$} & $f=1$ \\
\hline $0,6<x_{i}<0,7$ & & $f_{4}=10 *\left(0,7-x_{i}\right)$ \\
\hline $0,6<x_{i}<0,7$ & \multirow{2}{*}{$\begin{array}{l}\text { Alto y máximo valor de la variable, máxima calidad } \\
\text { de digitalización }\end{array}$} & $f_{5}=1-f_{4}$ \\
\hline $0,7<x_{i} \leq 1$ & & $f=1$ \\
\hline
\end{tabular}
subconjuntos [desarrollado por los autores]

El cálculo del coeficiente intermedio se realizó utilizando la fórmula (2), la evaluación final de la calidad de la digitalización está representada por la fórmula correspondiente (3), en la que los criterios de peso fueron especificados por la regla de Fishburn (Fórmula 4):

$$
\begin{aligned}
& k=\frac{\sum f_{i}}{n} \\
& F A=\sum k_{i} * w_{i} \\
& w=\frac{1}{N}
\end{aligned}
$$

En estas ecuaciones $k$ es el valor del coeficiente intermedio de la i-ésima función de pertenencia de una variable (parámetro) a un subconjunto; f es el valor de la i-ésima función de pertenencia de una variable (parámetro) a un subconjunto; $n$ es el número de parámetros de evaluación experta; $F A$ es el valor de la evaluación final de la calidad de la digitalización de las funciones de gestión universitaria.; $w$ es el peso del iésimo parámetro de la evaluación de los expertos; $N$ es la progresión aritmética de los factores que determinan el peso del i-ésimo parámetro (variable y su función de pertenencia) del juicio experto.

Así, los juicios lingüísticos y difusos los transformamos en estimaciones cuantitativas claras. En calidad de expertos, invitamos a participar en la encuesta a: i) 15 personas en el grupo de gestión de universidades en Rusia, Brasil, Chile, Argentina: estos expertos llevan a cabo funciones estrictamente gerenciales; ii) 35 personas del profesorado superior de las universidades que combinan funciones educativas y de investigación con funciones gerenciales; y iii) 15 personas del personal directivo y docente, que combinan en sus actividades todas las funciones indicadas en la Tabla 2. La lista de universidades que constituyen el principal lugar de trabajo de los expertos invitados se presenta en la Tabla 6. 
Tabla 5: Evaluación final de la calidad de digitalización de las funciones de gestión universitaria

\begin{tabular}{|c|c|c|}
\hline $\begin{array}{l}\text { Serie de valores para } \\
\text { la calificación final. }\end{array}$ & $\begin{array}{l}\text { Descripción del conjunto difuso de variables que caracterizan } \\
\text { la evaluación final de la calidad de la digitalización. }\end{array}$ & $\begin{array}{l}\text { Confianza en que la } \\
\text { calificación final pertenece } \\
\text { a un conjunto difuso }\end{array}$ \\
\hline $0<F A<0,15$ & \multirow{2}{*}{$\begin{array}{l}\text { La muy baja calidad de la digitalización de funciones de } \\
\text { gestión universitaria (y otras funciones interrelacionadas), lo } \\
\text { que no permite crear nuevas oportunidades para la } \\
\text { universidad y utilizar las posibilidades de desarrollo } \\
\text { emergentes }\end{array}$} & $f s=1$ \\
\hline $0,15<F A<0,25$ & & $f s_{1}=10 *(0,25-F A)$ \\
\hline $0,15<F A<0,25$ & \multirow{2}{*}{$\begin{array}{l}\text { La baja calidad de la digitalización de funciones de gestión } \\
\text { universitaria (y otras funciones interrelacionadas), lo que limita } \\
\text { significativamente la posibilidad de crear nuevas } \\
\text { oportunidades para la universidad y limita las posibilidades de } \\
\text { utilizar las oportunidades de desarrollo emergentes. }\end{array}$} & $f_{S_{2}}=1-f_{S_{1}}$ \\
\hline $0,25<F A<0,35$ & & $f_{S}=1$ \\
\hline $0,35<F A<0,45$ & \multirow{3}{*}{$\begin{array}{l}\text { Calidad de digitalización no superior al promedio o } \\
\text { posiblemente de calidad media de las funciones de gestión } \\
\text { universitaria (y otras funciones interrelacionadas), lo que limita } \\
\text { las posibilidades de crear nuevas oportunidades para la } \\
\text { universidad y limita las posibilidades de utilizar las } \\
\text { oportunidades de desarrollo emergentes }\end{array}$} & $f s_{2}=10 *(0,45-F A)$ \\
\hline $0,35<F A<0,45$ & & $f s_{3}=1-f s_{2}$ \\
\hline $0,45<F A<0,55$ & & $f s=1$ \\
\hline $0,55<F A<0,65$ & \multirow{2}{*}{$\begin{array}{l}\text { Calidad de digitalización promedio y ligeramente superior a la } \\
\text { media de las funciones de gestión universitaria (y otras } \\
\text { funciones interrelacionadas), lo que permite crear } \\
\text { oportunidades adicionales para la universidad, pero dificulta el } \\
\text { uso de las oportunidades de desarrollo emergentes }\end{array}$} & $f s_{3}=10 *(0,65-F A)$ \\
\hline $0,55<F A<0,65$ & & $f_{S_{4}}=1-f_{S_{3}}$ \\
\hline $0,65<F A<0,75$ & \multirow{2}{*}{$\begin{array}{l}\text { Calidad de digitalización de la gestión universitaria (y otras } \\
\text { funciones interrelacionadas) alta o superior a la media, lo que } \\
\text { permite crear oportunidades para la universidad y utilizar las } \\
\text { oportunidades de desarrollo emergentes }\end{array}$} & $f s=1$ \\
\hline $0,75<F A<0,85$ & & $f s_{4}=10 *(0,85-F A)$ \\
\hline $0,75<F A<0,85$ & \multirow{2}{*}{$\begin{array}{l}\text { La muy alta calidad de las funciones de gestión universitaria (y } \\
\text { otras funciones interrelacionadas), lo que permite crear } \\
\text { oportunidades únicas para la universidad y hacer un amplio } \\
\text { uso de las oportunidades de desarrollo emergentes. }\end{array}$} & $f s_{5}=1-f s_{4}$ \\
\hline $0,85<F$ & & $f s=1$ \\
\hline
\end{tabular}

Tabla 6: Lista de universidades, que constituyen el principal lugar de trabajo de los expertos invitados [compilado por los autores]

\begin{tabular}{|c|c|}
\hline País & Universidades \\
\hline \multirow[t]{8}{*}{ Rusia } & Universidad Estatal de los Urales del Sur \\
\hline & Universidad Estatal de los Urales \\
\hline & Universidad Federal del Norte \\
\hline & Universidad Minera de San Petersburgo \\
\hline & Universidad Estatal de Togliatti \\
\hline & Universidad Estatal "Dubna" \\
\hline & Universidad Estatal de Adygea \\
\hline & Universidad Estatal de Sajalín \\
\hline \multirow[t]{8}{*}{ Brasil } & Instituto Federal de Educación, Ciencia y Tecnología de S. Paulo \\
\hline & Universidad del Estado de Minas Gerais \\
\hline & Universidad de Tiradentes \\
\hline & Universidad Federal del Valle de San Francisco \\
\hline & Universidad del Amazonas \\
\hline & Centro Universitario de Vila Velha \\
\hline & Universidad de Sorocaba \\
\hline & Universidad Estatal de Santa Cruz \\
\hline \multirow[t]{5}{*}{ Chile } & Universidad de Magallanes \\
\hline & Universidad Pedro de Valdivia \\
\hline & Universidad La República \\
\hline & Universidad Bolivariana \\
\hline & Universidad del Pacífico \\
\hline \multirow[t]{7}{*}{ Argentina } & Universidad de Flores \\
\hline & Universidad de Mendoza \\
\hline & Universidad de Favaloro \\
\hline & Universidad Nacional de Chilecito \\
\hline & Universidad ISALUD \\
\hline & Universidad Nacional de Santiago del Estero \\
\hline & Instituto Universitario CEMIC \\
\hline
\end{tabular}



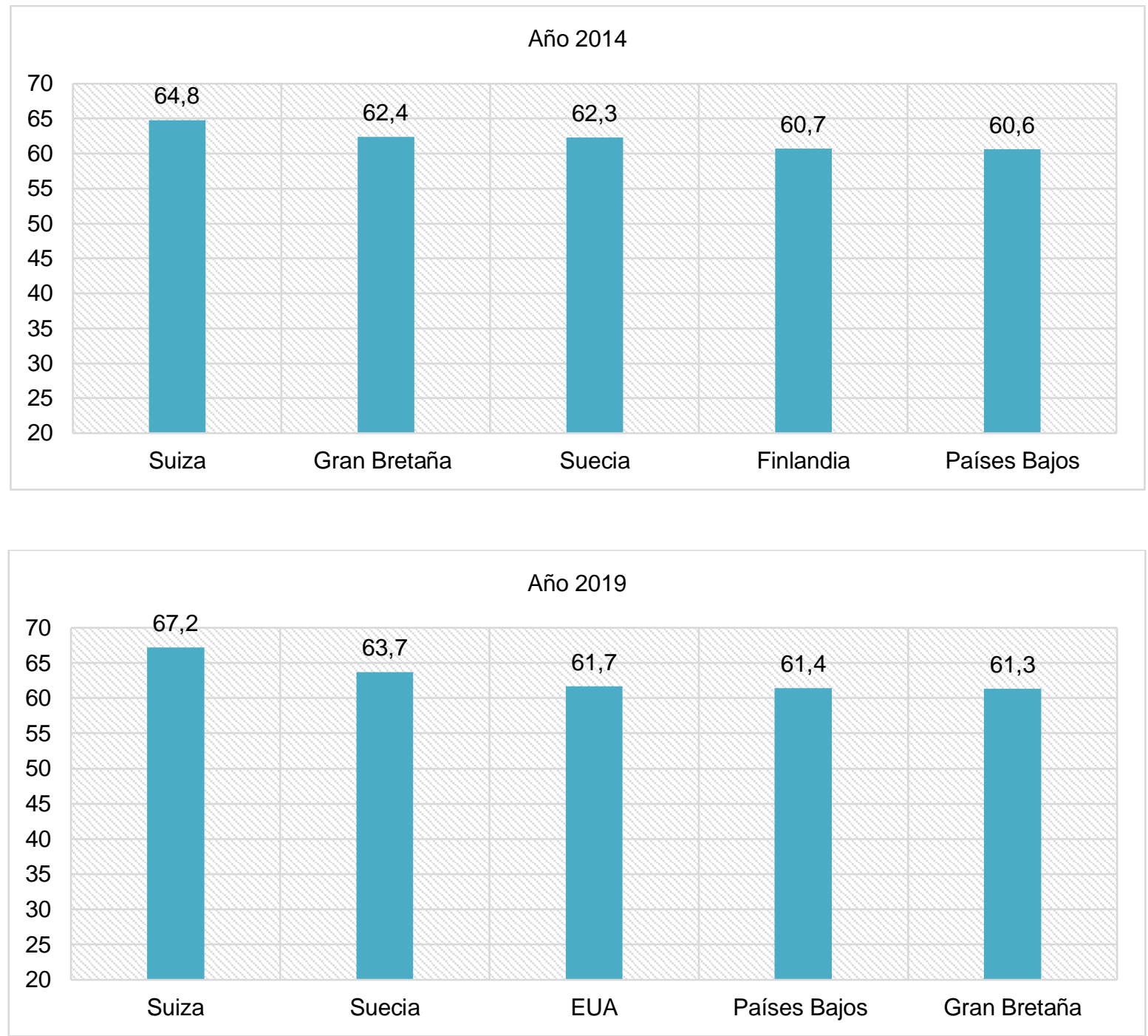

Fig. 1: Líderes en el ranking mundial por países según el índice de innovación (Global Innovation Index, 2019)

En el estudio no participaron expertos de las universidades principales de sus países, ya que, por regla general, las instituciones educativas y las organizaciones de investigación más importantes cuentan con el mejor nivel de financiación y el mejor apoyo logístico, incluido el tecnológico. En la encuesta participaron expertos de universidades que no figuran en la lista TOP-30 de las clasificaciones nacionales, lo que sugiere evaluaciones más relevantes del nivel de digitalización en la gestión de las universidades de Rusia, Brasil, Chile y Argentina.

\section{RESULTADOS}

Los líderes de la innovación moderna en 2019 fueron: Suiza, Suecia, EE.UU., los Países Bajos, Reino Unido y, en general, la situación en los últimos cinco años con respecto al TOP-5 es prácticamente la misma, los EE.UU. demuestran un éxito variable (Fig. 1). Estos países tienen todas las condiciones y recursos necesarios, para la innovación y muestran altos resultados la implementación de innovaciones. En total, de 130 a 140 países se han venido incluyendo en este ranking a través de los años. Los cuatro países analizados en este artículo (Rusia, Chile, Brasil, Argentina), por regla general, figuran fuera del mismo. (Fig. 2).

Antes que nada, se debe prestar atención al hecho de que todos los países analizados en este artículo han reducido su éxito de innovación: en Argentina y Chile, la disminución fue de aproximadamente un 10\%, en Rusia y Brasil en los últimos cinco años, aproximadamente de un $4 \%$ y un $7 \%$, respectivamente. La divergencia promedio con respecto al líder del ranking en el grupo de países analizados es de 24.5 a 32.5 puntos, lo que indica que: en el grupo de países analizados no existen condiciones para crear innovaciones (de hecho: no hay sistemas nacionales de innovación desarrollados); en el grupo de países analizados, la productividad de las innovaciones es muy baja (de hecho: no existe interacción entre la ciencia, el gobierno y las empresas para el desarrollo científico y de altas tecnologías). 


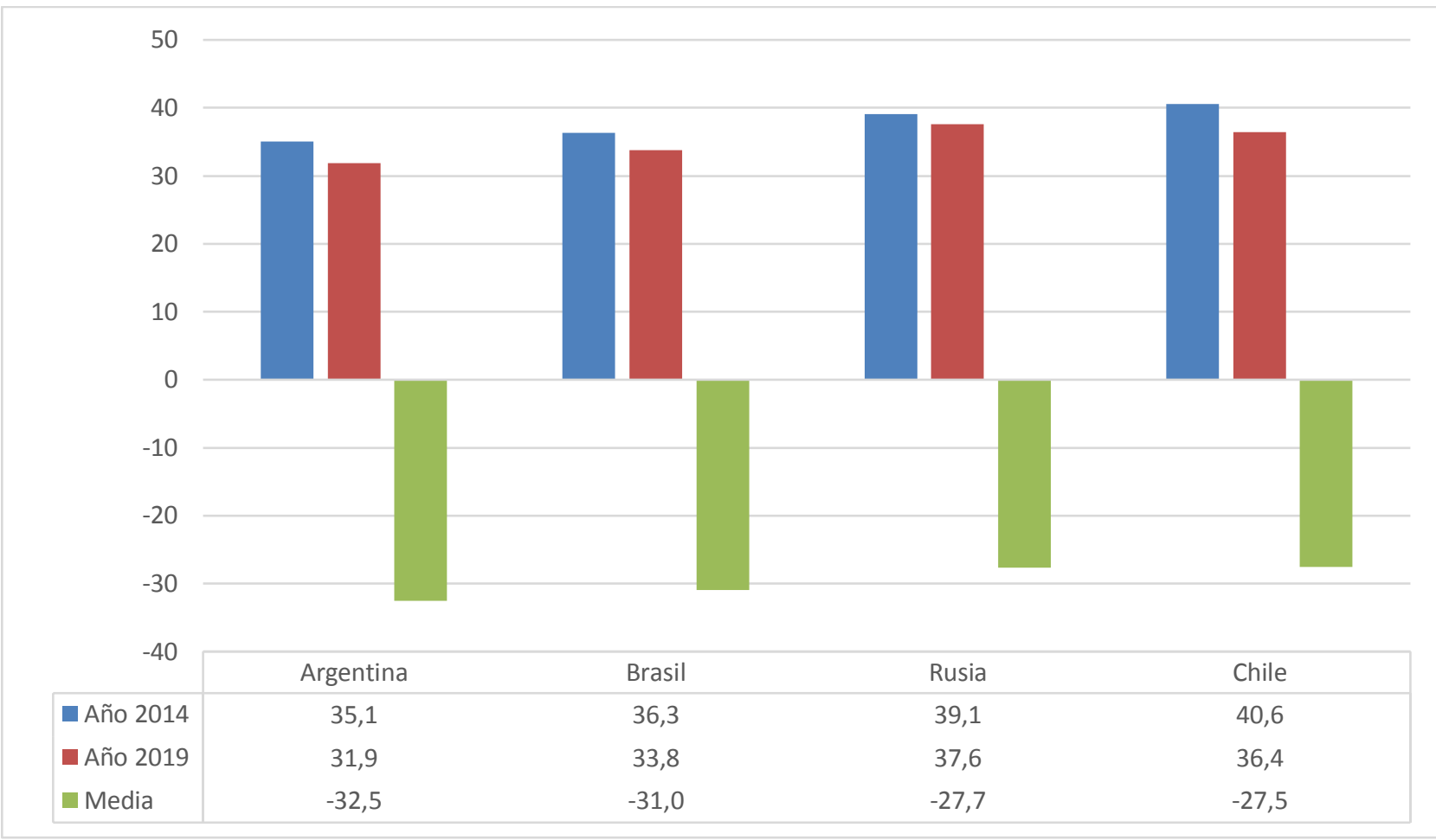

Fig. 2: Posiciones de los países analizados en el artículo en el ranking mundial de países por índice de innovación (Global Innovation Index, 2019). Media, significa Media de divergencia con respecto al líder

La innovación, la inversión, las instituciones con alta efectividad y la calidad de la educación deben considerarse componentes clave que dan forma al bienestar nacional. Si comparamos los tres componentes del sistema nacional de innovación (negocios, ciencia / educación y administración pública), podemos notar que los primeros lugares aquí serán ocupados por países que comúnmente se conocen como "tigres asiáticos" (Singapur y Hong Kong), así como países de Europa Occidental (Noruega, Finlandia, Dinamarca), Fig. 3.

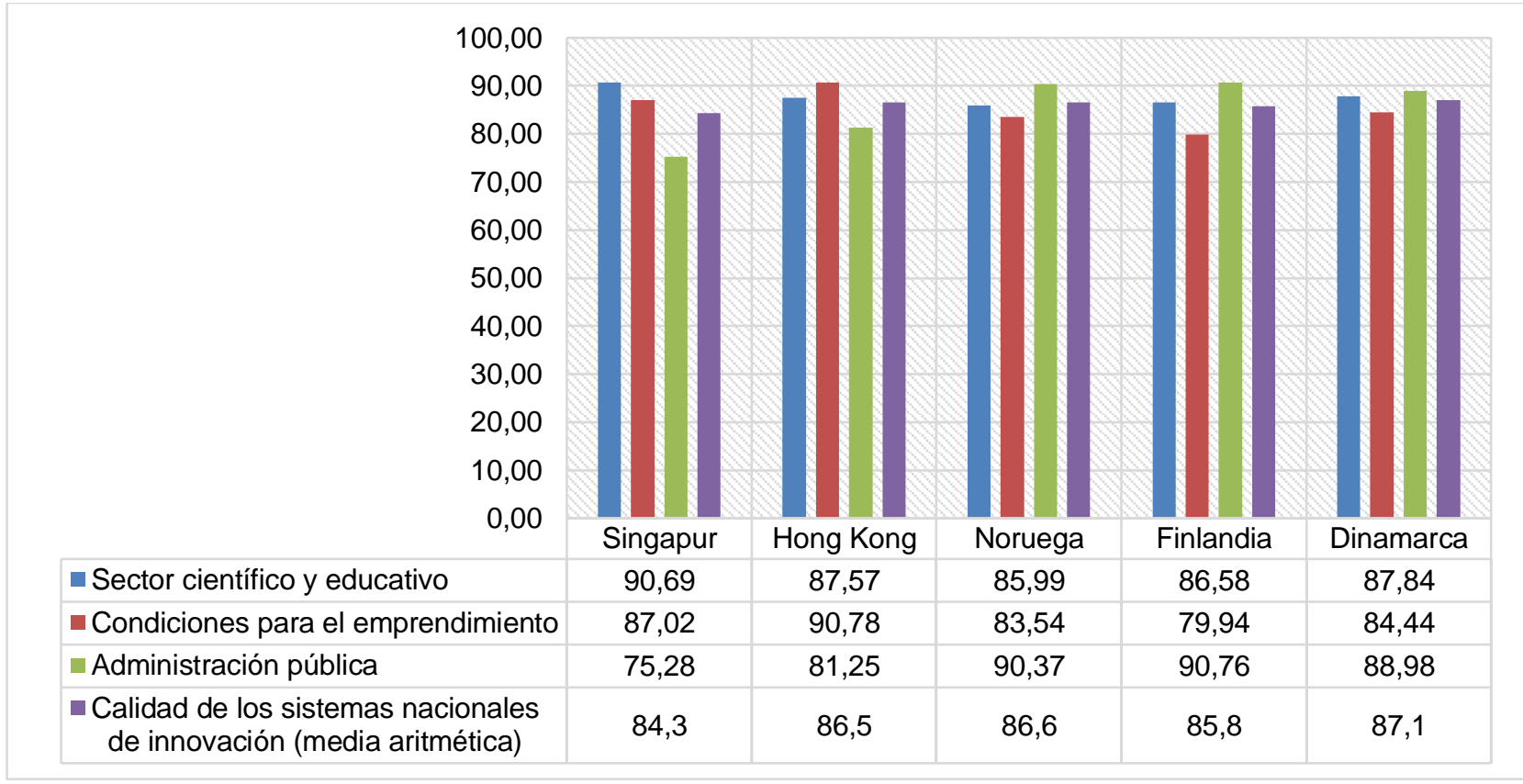

Fig. 3: Evaluación condicional del desarrollo de sistemas nacionales de innovación (NIS) basados en el modelo de la "Triple Helix" en Singapur, Hong Kong, Noruega, Finlandia, Dinamarca (The Legatum Prosperity Index, 2019)

Si comparamos indicadores similares para los cuatro países analizados (Fig. 4), podemos observar que Chile ha desarrollado las condiciones más favorables para la formación de un sistema nacional de innovación, en Rusia y Argentina solo el sector científico-educativo se ha desarrollado hasta el momento, y Brasil sigue figurando en posiciones externas. 


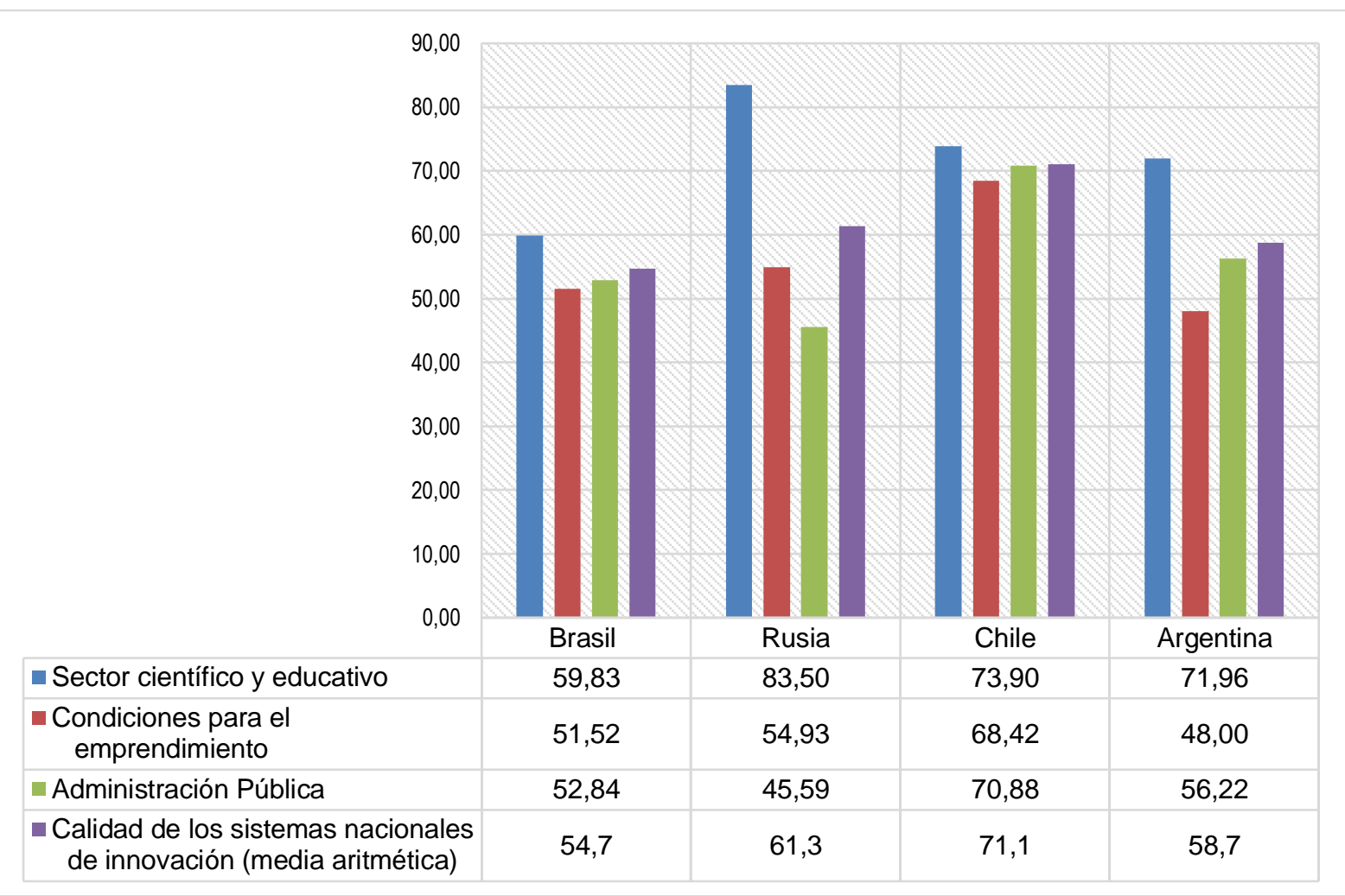

Fig. 4: Evaluación condicional del desarrollo de sistemas nacionales de innovación (NIS), el modelo "Triple Helix" en Rusia, Brasil, Chile y Argentina (The Legatum Prosperity Index, 2019)

Por lo tanto, en tres de los cuatro países se tienen todos los requisitos previos necesarios para la transición a una economía postindustrial, pero en realidad esto no sucede. Una de las causas de esto puede ser la ausencia de transición de los resultados de la actividad intelectual a sectores reales de la economía, que se produce debido a la mala o ausente comunicación entre el estado, la ciencia y el emprendimiento (negocios); esta situación es típica de Chile. En Rusia, Argentina y Brasil, la falta de comunicación en el modelo de triple hélice se ve complicada por las condiciones desfavorables para el emprendimiento (negocios) y por el bajo nivel de calidad de las funciones de Administración pública.

El problema puede resolverse parcialmente digitalizando la gestión del sector científico y educativo. Ya se ha demostrado anteriormente que 65 expertos estuvieron involucrados en el trabajo, sus juicios fueron generalizados por nosotros en la sección "Materiales y métodos". Los resultados de la conversión de opiniones de expertos sobre los principales grupos con el cálculo del coeficiente de concordancia se presentan en la Tabla 7.

Tabla 7: Análisis de la consistencia de las opiniones de expertos con respecto a la digitalización de la gestión universitaria en sus países [compilado y calculado por los autores]

\begin{tabular}{|l|l|l|l|}
\hline Factores (modelos variables de evaluación) & $\begin{array}{l}\text { Grupo de } \\
\text { expertos 1 }\end{array}$ & $\begin{array}{l}\text { Grupo de } \\
\text { expertos 2 }\end{array}$ & $\begin{array}{l}\text { Grupo de } \\
\text { expertos 3 }\end{array}$ \\
\hline $\begin{array}{l}\text { Competencias necesarias para la digitalización de la } \\
\text { gestión universitaria, } x_{1}\end{array}$ & 0,55 & 0,48 & 0,51 \\
\hline $\begin{array}{l}\text { Herramientas y equipos necesarios para digitalizar la } \\
\text { gestión universitaria, } x_{2}\end{array}$ & 0,25 & 0,31 & 0,33 \\
\hline $\begin{array}{l}\text { Preparación para el cambio: transición a la digitalización } \\
\text { de la gestión universitaria, } x_{3}\end{array}$ & 0,4 & 0,4 & 0,34 \\
\hline $\begin{array}{l}\text { Financiación de la digitalización de la gestión universitaria, } \\
x_{4}\end{array}$ & 0,48 & 0,49 & 0,35 \\
\hline $\begin{array}{l}\text { Beneficios y perspectivas que aportaría la digitalización de } \\
\text { la gestión universitaria, } x_{5}\end{array}$ & 0,73 & 0,75 & 0,79 \\
\hline Coeficiente de concordancia & $W=0,956 ;$ alta convergencia de opiniones \\
\hline $\begin{array}{l}\text { Valor del coeficiente de concordancia según el criterio de } \\
\text { Pearson }\end{array}$ & $\begin{array}{l}\square^{2}=11,47<9,48773 ; \text { alto valor del coeficiente, } W \text { no } \\
\text { es una variable aleatoria }\end{array}$ \\
\hline
\end{tabular}


Los juicios de expertos obtenidos se pueden formular de la siguiente manera: en primer lugar, todos los expertos entrevistados están de acuerdo en que la digitalización de la gestión proporciona a la universidad importantes ventajas competitivas y abre nuevas áreas prometedoras de desarrollo; en segundo lugar, todos los expertos opinan que el nivel de competencias necesario para realizar el cambio a la digitalización de la gestión universitaria de los dirigentes universitarios y del profesorado superior es medio; en tercer lugar, todos los expertos evalúan la financiación de la digitalización de la gestión universitaria como insuficiente, superior al mínimo, pero inferior a la media. Es similar la evaluación de la disposición para el cambio.

Teniendo en cuenta la opinión generalizada de los expertos con respecto a la importancia de la digitalización de la gestión de universidades y su desarrollo no en las principales universidades de los cuatro países analizados (Rusia, Brasil, Chile, Argentina), realizamos una evaluación final de la calidad de la digitalización utilizando un modelo múltiple difuso. Anteriormente, los expertos de cada país expresaron su opinión sobre la calidad de la digitalización de las funciones de gestión universitaria, basándose en su conocimiento y experiencia previa en este campo. Los datos obtenidos se procesaron utilizando el método de cálculo del valor medio geométrico, los resultados para cada país se presentan en la Tabla 8.

Tabla 8: Valores de las variables incluidas en el modelo difuso-plural para evaluar la calidad de la digitalización gerenciales y funciones vinculadas a estas en universidades de Rusia, Chile, Brasil, Argentina [compiladas y calculadas por los autores]

\begin{tabular}{|l|l|l|l|l|}
\hline \multirow{2}{*}{ Variables, incluidas en el modelo } & \multicolumn{4}{|l|}{$\begin{array}{l}\text { Estimación promedio geométrica de las variables establecidas } \\
\text { por expertos en sus países. }\end{array}$} \\
\cline { 2 - 6 } & Rusia & Brasil & Chile & Argentina \\
\hline $\begin{array}{l}\text { Competencias requeridas para digitalizar la gestión } \\
\text { universitaria }\end{array}$ & 0,53 & 0,48 & 0,49 & 0,55 \\
\hline $\begin{array}{l}\text { Herramientas y equipos necesarios para digitalizar la } \\
\text { gestión universitaria. }\end{array}$ & 0,25 & 0,25 & 0,33 & 0,34 \\
\hline $\begin{array}{l}\text { Disposición para el cambio: transición a la } \\
\text { digitalización de la gestión universitaria }\end{array}$ & 0,38 & 0,37 & 0,42 & 0,43 \\
\hline $\begin{array}{l}\text { Financiación de la digitalización de la gestión } \\
\text { universitaria }\end{array}$ & 0,55 & 0,49 & 0,51 & 0,55 \\
\hline $\begin{array}{l}\text { Beneficios y perspectivas de la digitalización de la } \\
\text { gestión universitaria }\end{array}$ & 0,76 & 0,71 & 0,75 & 0,79 \\
\hline
\end{tabular}

Además, todas las variables con sus funciones de membresía se ponderaron utilizando la regla Fishburn, que permitió establecer su peso para cada variable y la función correspondiente. Luego, utilizando las funciones matemáticas de pertenencia y confianza, se realizó una evaluación de la calidad de la digitalización de las funciones de gestión universitaria en cada uno de los cuatro países considerados, los resultados del cálculo se muestran en la Tabla 9.

Tabla 9: Evaluación final de la calidad de digitalización de funciones gerenciales y relacionadas en universidades de Rusia, Chile, Brasil, Argentina [compilada y calculada por los autores]

\begin{tabular}{|c|c|c|c|c|c|}
\hline \multirow{2}{*}{ Variables incluidas en el modelo } & \multicolumn{5}{|c|}{ Valor de la función de pertenencia de la variable. } \\
\hline & $f\left(x_{i}\right)_{1}$ & $f\left(x_{i}\right)_{2}$ & $f\left(x_{i}\right)_{3}$ & $f\left(x_{i}\right)_{4}$ & $f\left(x_{i}\right)_{5}$ \\
\hline \multicolumn{6}{|l|}{ Rusia } \\
\hline Variable $x_{1}$ & 0,00 & 0,00 & 0,20 & 0,80 & 0,00 \\
\hline Variable $x_{2}$ & 0,00 & 1,00 & 0,00 & 0,00 & 0,00 \\
\hline Variable $x_{3}$ & 0,00 & 0,00 & 1,00 & 0,00 & 0,00 \\
\hline Variable $x_{4}$ & 0,00 & 0,00 & 0,00 & 1,00 & 0,00 \\
\hline Variable $x_{5}$ & 0,00 & 0,00 & 0,00 & 0,00 & 1,00 \\
\hline Coeficiente intermedio & 0,00 & 0,20 & 0,24 & 0,36 & 0,20 \\
\hline Valor de peso & 0,93 & 0,31 & 0,50 & 0,72 & 0,07 \\
\hline Valor de la evaluación de calidad de la digitalizació & \multicolumn{5}{|c|}{0,455} \\
\hline \multicolumn{6}{|c|}{ Interpretación del resultado utilizando la función de confianza. } \\
\hline \multicolumn{4}{|c|}{$\begin{array}{l}\text { Calidad no superior al promedio o condicionalmente promedio de la digitalización gerencial y funciones vinculadas } \\
\text { a ésta. }\end{array}$} & \multicolumn{2}{|c|}{$54,5 \%$} \\
\hline \multicolumn{4}{|c|}{ Calidad media o ligeramente superior a la media de la digitalización gerencial y funciones vinculadas a ésta. } & \multicolumn{2}{|c|}{$45,5 \%$} \\
\hline
\end{tabular}


Tabla 9: continuación

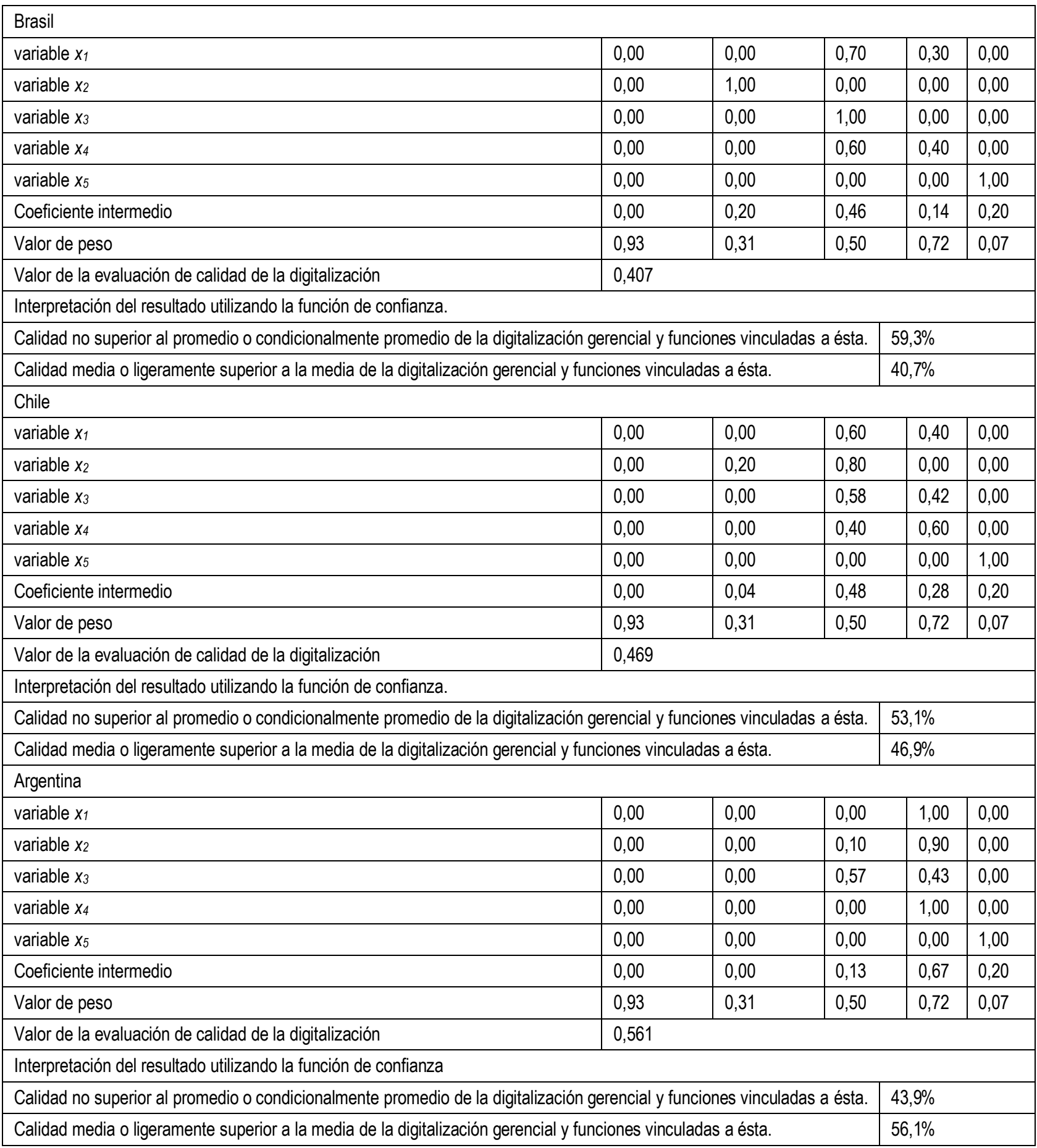

Entonces, los resultados de la evaluación muestran que, en Rusia, Brasil, Chile y Argentina, la calidad de la digitalización de las funciones gerenciales y de otro tipo en las universidades es aproximadamente igual. El valor estimado más bajo se obtuvo para Brasil, el más alto para Argentina, mientras que la dispersión de valores es muy baja. Esto nos permite concluir que las tendencias y patrones de digitalización de los sistemas nacionales de ciencia y educación en los cuatro países considerados serán aproximadamente iguales.

\section{DISCUSIÓN}

Como se puede ver en los materiales presentados para el análisis, las universidades de Rusia y de los países latinoamericanos seleccionados siguen poniéndose al día en las tendencias del desarrollo global de la digitalización de la gestión de las instituciones de educación superior. Hoy se pueden destacar algunos problemas y contradicciones comunes que obstaculizan el desarrollo posterior de la digitalización de la gestión de universidades, tanto en Rusia como en los países seleccionados de América del Sur. 
El modelo organizativo y financiero de gestión de universidades con una gran participación del estado, que era y sigue siendo un propietario no lo suficientemente efectivo, no es consistente, en general, con la idea de promover la innovación. El objetivo principal del estado y la sociedad es garantizar las mayores oportunidades para obtener una educación superior accesible. Esta accesibilidad está en conflicto con la efectividad económica (financiera) del funcionamiento de las universidades. La posesión colectiva de las universidades, que se ha generalizado bastante en la práctica latinoamericana, tampoco dispone por completo la intensificación de las innovaciones, ya que los mecenas privados como los padres de los estudiantes (reales o futuros) están interesados en precios bajos y alta disponibilidad de educación, a menudo incluso a expensas de la calidad. Cabe señalar que en Europa y América del Norte, los líderes en digitalización no son simplemente universidades privadas, sino que, por regla general, están estrechamente integradas en el sistema de posesión de grandes corporaciones, o financiadas por estas corporaciones.

La administración y los propietarios de corporaciones fueron los primeros, de hecho, en apreciar los beneficios de la digitalización de la gestión de procesos comerciales, sobre la base de la cual, comenzaron a realizar mejoras en todas las áreas potenciales (Schwertner, 2017; Viryasitavat et al., 2018). La situación, que permita a la práctica rusa de gestión de la digitalización alcanzar un nivel de desarrollo notable a escala mundial (Drozdova y Petrov, 2018), puede considerarse parcialmente solo como una paradoja; la explicación principal es la autonomía significativa en la administración de regiones individuales, en combinación con enormes recursos financieros, asignados para resolver el problema de optimización en este caso. El sistema de universidades no tiene tales capacidades.

La falta de práctica avanzada de gestión de cambios en la universidad, sobre la base de un paradigma de calidad. En Rusia recientemente comenzaron a clasificar las actividades de universidades como las de servicio, analizándolas exclusivamente desde la posición del sistema social (Babin, 2018). Representantes de universidades latinoamericanas entrevistados por nosotros, señalan que aquí también se plasman ideas sobre la orientación exclusivamente social de la percepción del servicio universitario; la gestión del servicio educativo se lleva a cabo partiendo de la solicitud pública y no de la calidad o de la competitividad;

La interacción insuficiente por "triple hélice". Actualmente, uno de los paradigmas principales del desarrollo de una economía innovadora es el concepto de "triple hélice", según el cual, a través de la estrecha integración del estado, las empresas y las universidades se crea un entorno en el que se aseguran condiciones favorables para el desarrollo de innovaciones (Geoghegan et al., 2015; Etzkowitz y Zhou, 2017) (Hoy, al mismo tiempo, el vector de innovación está asociado en gran medida a la digitalización). Si no se ha creado "triple hélice", se forma la paradoja de "doble nudo de opresor" [concepto de los autores]: por un lado, no existen requisitos institucionales y ambientales para el desarrollo de la digitalización como tal; por otro lado, las universidades no están suficientemente integradas en el intercambio de innovación y tecnología con otros participantes en la "hélice", y no solo no contribuyen a la creación y desarrollo de innovaciones, sino que también, están extremadamente limitadas en su capacidad de hacer uso de los resultados;

El vector de interés práctico en la digitalización, dirigido al proceso educativo, en detrimento de su administración. En otras palabras, se están desarrollando cuestiones relacionadas con la digitalización de la enseñanza, desde instrumentaciones como plataformas digitales para la educación a distancia, hasta aplicaciones móviles para estudiantes y profesores. Tales innovaciones se relacionan con la digitalización de manera indirecta, podrían llamarse lite-digitalización. Se están realizando proyectos más serios, en áreas como el uso de la inteligencia artificial, sistemas de autoaprendizaje, en particular, la red neuronal, en la formación de planes de estudio, e incluso en la preparación de cursos. Estamos hablando de hard digitalización, pero los desarrolladores no pueden compartir éxitos significativos con las universidades en la etapa actual. Al mismo tiempo, en la digitalización de la gestión, las administraciones de universidades pueden usar soluciones "preparadas" del entorno empresarial y corporativo, pero no lo hacen, en parte, debido a las interacciones ineficientes de "triple hélice"; La falta de soluciones de "paquete" sectoriales para digitalizar los procesos comerciales de la gestión de universidades, incluidas las plataformas de gestión integradora y las soluciones aplicadas para digitalizar la planificación, el control, etc.

Uno de los problemas para la ulterior digitalización de la gestión en las universidades modernas, es la insuficiente competencia digital del personal. En realidad, si hablamos de Rusia, por ejemplo, entonces la gestión del personal de las universidades por competencias es una nueva dirección, que no está lo suficiente probada en todos los aspectos. Como regla general, solo unas pocas universidades más grandes de nivel federal, introducen elementos de gestión de competencias, y esta gestión, hasta ahora solo abarca ciertos grupos de profesores, y rara vez es aplicada en la arquitectura general de gestión del desarrollo universitario. Las encuestas a representantes de universidades en América del Sur, indican un problema similar: en ninguna de las universidades examinadas se estudian los problemas de competencia digital del personal administrativo y, por lo tanto, no se plantea la tarea de su formación y desarrollo. Incluso si tener en cuenta el hipotético alto 
nivel de cultura digital de los profesores, que, obviamente, no resuelve el problema, ya que el personal administrativo desarrolla e implementa directamente soluciones específicas;

Además, nos gustaría señalar la existencia de un excesivo ruido informativo alrededor de la digitalización en el entorno de gestión científica, cuya presencia, como creemos, en sí misma reprime la digitalización de la gestión, especialmente en un entorno socialmente orientado. En resumen, este ruido consiste en que los investigadores prefieren limitarse a las declaraciones sobre la necesidad de digitalización, pero en el futuro, el estudio es limitado a la teorización, sin entrar en un plano práctico. Nuestra encuesta a los representantes de las administraciones de las universidades rusas y latinoamericanas confirmó la importancia de los problemas identificados y su influencia incondicional en el atraso del sistema universitario nacional con respecto a los líderes mundiales. Las insignificantes desviaciones de los resultados obtenidos por los expertos, más bien, confirman las limitaciones metodológicas bien conocidas que ponen en duda los resultados obtenidos; en otras palabras, los trabajadores prácticos confirman, tanto la presencia como la importancia de los problemas y contradicciones correspondientes.

En consecuencia, la situación con la digitalización de la gestión de universidades en Rusia y los países de América Latina seleccionados para el estudio, se caracteriza por numerosos aspectos comunes, lo que sugiere la posibilidad y la validez de implementar soluciones universales adecuadas para implementación y puesta en práctica. Para garantizar soluciones en la digitalización de la gestión de universidades, basándose en la experiencia de las universidades de los países líderes y en los elementos de la experiencia acumulada de las universidades en Rusia y América Latina, parece apropiado implementar un sistema con las siguientes medidas:

En primer lugar, seleccionar prometedoras instrumentaciones de digitalización, principalmente basándose en aquellas, cuya implementación es posible en la etapa actual, entre ellas, las que se refieren al desarrollo de tecnologías, incluyendo sus pruebas exitosas en el sector comercial de la economía. Como tales, pueden denominarse la planificación digital y el control digital, partiendo de las funciones clave de gestión como ciencia. La digitalización de la organización, a su vez, tiene mayor prioridad para la tarea particular de digitalizar el proceso educativo (el proceso de proveer servicios educativos).

Como una parte de la planificación, en nuestra opinión, las plataformas digitales para la planificación integradora de las actividades universitarias, tienen perspectivas significativas. La idea principal es, con la ayuda de la digitalización, que combinará planes de todos los niveles, integrar la planificación de los recursos claves de la universidad, unir la planificación estratégica, operativa y táctica, asegurar una gestión más efectiva de los recursos limitados de la universidad; y al mismo tiempo, tener en cuenta la identificación oportuna y la consideración de los riesgos internos y externos.

La auditoría y el control digital están basados en un enfoque de riesgo. La idea principal es, que con el uso de instrumentación de digitalización, es posible no solo reducir los riesgos de impacto del factor humano en los procesos y procedimientos de control, sino también aumentar significativamente su efectividad, al digitalizar los procesos preparatorios, en particular, la identificación de las áreas de gestión más problemáticas, que requieren un control más atento.

Otra área prometedora de la digitalización es el uso de instrumentación de big data para desarrollar y planificar servicios y procesos comerciales de la universidad, basada en la integración de la administración digital y la digitalización de servicios educativos. Entonces, en cierta medida, con el uso de la tecnología para analizar big data, hoy ya existe la posibilidad de crear un sistema que rastrea el comportamiento de los estudiantes en el proceso educativo, y pasar a la introducción de sistemas inteligentes de gestión de procesos educativos. Con el uso de las nuevas tecnologías, es posible tomar una "huella digital del estudiante", y conocer, cuándo el estudiante abrió el libro de texto, cuántos segundos miró y escuchó cada página, cómo respondió a cada pregunta de la prueba, si leyó el libro de texto una vez más en caso de falla. Por estos datos del proceso educativo es posible construir una tipología de los comportamientos educativos de los estudiantes, y a cada estudiante ofrecer apoyo en el proceso educativo, que le permita mostrar los máximos resultados posibles de aprendizaje, y así construir un sistema de aprendizaje verdaderamente adaptativo, y desde la posición de los consumidores de servicios educativos, garantizar el máximo de conformidad del servicio educativo con las expectativas racionales de los consumidores: estudiantes, sus padres y posibles empleadores; en segundo lugar, planificar y garantizar la implementación sistemática de proyectos de digitalización, sin priorizar un área específica de gestión o una tecnología digital específica. La digitalización total de los procesos de gestión de una universidad moderna debe estar precedida por varias etapas de transición, comenzando por el establecimiento inicial de objetivos, el desarrollo de programas y de los planes correspondientes, los proyectos tecnológicos y de otro tipo, e incluyendo la etapa más importante de transformación digital, que implica la reingeniería de los procesos comerciales sobre la base de tecnologías digitales implementadas; en tercer lugar, garantizar la gestión apropiada del personal administrativo y del profesorado de las universidades, sobre 
la base de un enfoque de competencias, y prestando especial atención a la gestión de la competencia digital de los participantes en el proceso de aprendizaje y el proceso de administración de la universidad.

En particular, un requisito importante al personal de una universidad moderna es un alto nivel de competencia digital; lo anterior especialmente se refiere al personal administrativo, que actuará como conductor de la digitalización de la gestión de la universidad. La gestión por competencias debe convertirse en la dirección fundamental de la gestión de recursos humanos de la universidad, con la priorización de las competencias digitales, su evaluación y desarrollo. Los planes para el desarrollo individual del personal administrativo, la capacitación profesional, etc. deben estar subordinados a la tarea de aumentar la competencia digital integrada del personal de la universidad. Por supuesto, en el proceso de cambios, tendremos que enfrentarnos con la resistencia de la organización, especialmente relacionada con los temores de que durante las innovaciones "la máquina destruirá a la persona". En consecuencia, además de la instrumentación estándar para superar la resistencia a los cambios, como informar, motivar, trabajar con líderes de opinión, implementar soluciones de digitalización para la gestión de la universidad, se debe tener un plan para resolver los problemas de aseguramiento de empleo del personal liberado, pues muy a menudo, esto entra dentro de los requisitos imperativos del gobierno. Cuando se desarrollan programas y planes apropiados, entre otras cosas, parece razonable guiarse por la consideración de que el personal administrativo liberado, como resultado, puede ser redirigido de forma eficiente a la gestión de sistemas inteligentes, y en algunos casos esto no requerirá esfuerzos significativos de capacitación;

En cuarto lugar, asegurar la gestión apropiada de los riesgos relacionados con la digitalización de los procesos de gestión. Hablamos sobre algunos de ellos anteriormente, incluso los riesgos de resistencias organizacionales, así como los problemas socialmente significativos de integrar los gerentes liberados en el mercado laboral actualizado. Sin embargo, la digitalización, garantizada por la automatización (el uso de inteligencia artificial), también conlleva riesgos informáticos específicos, como riesgos de ininterrumpido funcionamiento tecnológicamente ajustado, riesgos de integridad de la información y su protección contra interferencias externas.

Mientras mayor sea el nivel de digitalización de la gestión, mayores serán no solo la probabilidad de que aparezcan los riesgos correspondientes, sino también el daño potencial como consecuencia de estos. Por esta razón, el desarrollo de soluciones en la digitalización de la gestión de universidades debe estar acompañado de la implementación de medidas extendidas para prevenir riesgos y amenazas a la seguridad, desde la planificación basada en un enfoque de riesgo, hasta la implementación de un sistema corrector y compensador de impactos;

En quinto lugar, crear presupuestos para multiplicar los conocimientos técnicos en la digitalización, en el marco del sistema educativo nacional. No para cerrar, sino, por el contrario, para revelar innovaciones. Colaborar con otras universidades, empresarios y con el estado en el desarrollo de soluciones tecnológicas ("en caja") para la digitalización.

A un nivel macroeconómico, en paralelo con las medidas presentadas, se expone como objetivo, garantizar una solución efectiva y oportuna de las siguientes tareas prioritarias, que contribuyen a crear un entorno para la digitalización exitosa de la gestión de universidades, y que tienen en cuenta el funcionamiento del sistema nacional de educación superior, así como las características específicas del desarrollo socioeconómico: en primer lugar, proveer asistencia integral y apoyo por parte del estado a empresas relacionadas con la digitalización de la gestión de universidades. Por ejemplo, este apoyo puede expresarse en la cofinanciación por el estado del desarrollo de plataformas para digitalizar la gestión de universidades, en la transferencia de tecnologías, conocimientos, creación de grupos de trabajo, etc.; en segundo lugar, activar la solución del problema de construir una "triple hélice" de interacción en una economía innovadora.

Al mismo tiempo, los materiales de algunos estudios de los resultados (incluso los intermedios) de la digitalización de la gestión en las principales universidades de la UE, EE. UU. y Canadá (Haukijärvi, 2016; Pathak, 2016) indican que: en primer lugar, la digitalización es la base principal para los cambios cualitativos en el sistema de gestión de universidades, lo que permite depurar procesos comerciales y crear presupuestos excepcionales para la gestión digital del proceso educativo; en segundo lugar, la digitalización permite asegurar la gestión más eficiente de los recursos universitarios, que es inaccesible si se utilizan métodos e instrumentación tradicionales; en tercer lugar, la digitalización garantiza los presupuestos para asegurar y apoyar la competitividad local, nacional y global de los servicios educativos, que no pueden ser menos importantes desde un punto de vista estratégico, que los éxitos intermedios en forma de mejoras (tanto situacionales como estables a largo plazo), KPI financieros y organizacionales.

Por lo tanto, la digitalización de la gestión, en su esencia, se centra eminentemente en garantizar un impacto positivo en la competitividad de los servicios educativos, su calidad; y el desarrollo estable, dinámico y 
equilibrado de las universidades. Obviamente, la digitalización de la gestión es una clave importante para la formación exitosa de la universidad del futuro, y como tal formación ya está teniendo lugar, cualquier retraso irrazonable en la preparación e implementación de proyectos de digitalización de la administración universitaria, puede causar enormes diferencias entre los líderes universitarios en un futuro muy cercano. Para las economías emergentes rusas y de los países latinoamericanos seleccionados, la tarea de digitalización de la gestión de la educación superior, en vista de lo anterior, adquiere un nivel de seguridad económica nacional, ya que un entorno universitario desarrollado, su interacción integradora con el estado y los empresarios, se considera una garantía de restauración (si corresponde), preservación e intensificación de crecimiento económico, bienestar de la población, competitividad en los mercados mundiales de productos y servicios, así como también en el mercado laboral mundial, lo que constituye el interés de todos.

\section{CONCLUSIONES}

En el presente artículo analizamos el estado de la digitalización de las funciones de gestión (incluidas las funciones vinculadas entre sí) en universidades de Rusia, Brasil, Chile y Argentina. Los resultados obtenidos, en primer lugar, indican que, en estos cuatro países, los sistemas nacionales de innovación están en proceso de desarrollo cualitativo, es decir, no son suficientemente productivos. En segundo lugar, la calidad de la digitalización de la gestión universitaria en Rusia, Brasil, Chile y Argentina se encuentra cerca del nivel promedio, a pesar del hecho de que tanto el personal directivo como el profesorado es consciente de la importancia, la necesidad y la conveniencia de la transición a las tecnologías digitales de gestión. En resumen, el estudio confirma la hipótesis de que existe una relación objetiva entre el nivel de sofisticación de los sistemas nacionales de innovación y la calidad de la digitalización de las funciones de gestión universitaria.

\section{REFERENCIAS}

Babin, E.N., Digitalization of the university: construction of the integrated informational environment, https://doi.org/10.15826/umpa.2018.06.057, University Management: Practice and Analysis, 22(6), 44-54 (2018)

Bogoviz, A.V., Gimelshteyn, A.V., y otros tres autores, Digitalization of the Russian education system: Opportunities and perspectives, Quality-Access to Success, ISSN: 1582-2559, 19(S2), 27-32 (2018)

Brennen, J.S., y Kreiss, D., Digitalization, https://doi.org/10.1002/9781118766804.wbiect111, The international encyclopedia of communication theory and philosophy, 1-11 (2016)

Drozdova, I., y Petrov, A., World practice and Russian experience of housing and utilities sector digitization, https://doi.org/10.1051/shsconf/20184400031, SHS Web of Conferences, EDP Sciences, 44 (2018)

Dudin, M., Bezbakh, V., Frolova, E., y Galkina, M. Models of the higher education in Russia and the countries of Europe at the beginning of the 21st century: main directions of development, https://doi.org/10.13187/ejced.2018.4.653, European Journal of Contemporary Education, 7(4), 653-667 (2018)

Etzkowitz, H., y Zhou, C., The triple helix: University-industry-government innovation and entrepreneurship, https://doi.org/10.4324/9781315620183, Routledge (2017)

Geoghegan, W., O'Kane, C., y Fitzgerald, C., Technology transfer offices as a nexus within the triple helix: the progression of the university's role, https://doi.org/10.1504/IJTM.2015.069660, International Journal of Technology Management, 68(34), 255-277 (2015)

Global Innovation Index, https://www.globalinnovationindex.org/analysis-indicator (2019)

Godin, B., National innovation system: The system approach in historical perspective, https://doi.org/10.1177/0162243908329187, Science, Technology, \& Human Values, 34(4), 476-501 (2009)

Hattinger, M., Engeström, Y., y Sannino, A., From contradictions to transformation: a study of joint Work-Integrated Elearning between Industry and University, ISSN: 0096-0640, Journal of Engineering Education (2018)

Haukijärvi, I., Strategizing Digitalization in a Finnish Higher Education Institution: Towards a thorough strategic transformation, ISSN: 1455-1616, Acta Universitatis Tamperensis 2181, Tampere University Press (2016)

Legner, C., Eymann, T., y otros siete autores, Digitalization: opportunity and challenge for the business and information systems engineering community, https://doi.org/10.1007/s12599-017-0484-2, Business \& information systems engineering, 59(4), 301-308 (2017)

Leydesdorff, L., y Etzkowitz, H., Emergence of a Triple Helix of university—industry—government relations, https://doi.org/10.1093/spp/23.5.279, Science and public policy, 23(5), 279-286 (1996)

Maymina, E., Puzynya, T., y Egozaryan, V., Development trends of the education in Russia under digital economy, Revista ESPACIOS, ISSN: 0798-1015, 39(30) (2018)

$\mathrm{Ng}, \mathrm{W} .$, New Digital Technology in Education, Springer International PU (2016)

Ordorika, I., y Rodríguez-Gómez, R., Field of higher education research in Latin America, https://doi.org/10.1007/978-94017-9553-1_177-2, Encyclopedia of International Higher Education Systems and Institutions, Springer, Países Bajos (2018) 
Pathak, B.K., Emerging online educational models and the transformation of traditional universities, https://doi.org/10.1007/s12525-016-0223-4, Electronic Markets, 26(4), 315-321 (2016)

Schwertner, K., Digital transformation of business, https://doi.org/10.15547/tjs.2017.s.01.065, Trakia Journal of Sciences, 15(1), 388-393 (2017)

Slobodskaya, I.I., Analysis of the activities of resident organizations of the hi-tech Park of the Republic of Belarus, Banking Business and Financial Economy: Modern State, Global Trends and Prospects of Development, 261-264 (2018)

Strack, H., Wefel, S., y otros ocho autores. eID \& eIDAS at University Management-Chances and Changes for Security \& legally Binding in cross boarder Digitalization, https://doi.org/10.17879/21299722960, Proceedings of EUNIS, Universität Münster, 133-142 (2017)

The Legatum Prosperity Index, https://www.prosperity.com/rankings (2019)

United Nations, About the Sustainable Development Goals, https://www.un.org/sustainabledevelopment/sustainabledevelopment-goals/ (2020)

Vuori, V., Helander, N., y Okkonen, J., Digitalization in knowledge work: the dream of enhanced performance, https://doi.org/10.1007/s10111-018-0501-3, Cognition, Technology \& Work, 21(2), 237-252 (2019) 